\title{
Investigation and Analysis of Sharp Injuries among Health Care Workers from 36 Hospitals in Shandong Province, China
}

\author{
Jian Sun, ${ }^{1}$ Wen Qin, ${ }^{2}$ Lei Jia, ${ }^{1}$ Zhen Sun, ${ }^{1}$ Hua Xu, ${ }^{1}$ Yiyi Hui, ${ }^{1}$ Anman Gu, \\ and Weiguang $\mathbf{L i}$ (i) \\ ${ }^{1}$ Department of Infection Control, Shandong Provincial Hospital Affiliated to Shandong First Medical University, Jinan, \\ Shandong 250021, China \\ ${ }^{2}$ Department of Infection Control, The Affiliated Hospital of Qingdao University, Qingdao 266062, China
}

Correspondence should be addressed to Weiguang Li; emlwg@sina.com

Received 8 May 2020; Accepted 5 June 2021; Published 12 June 2021

Academic Editor: Mihajlo Jakovljevic

Copyright ( 2021 Jian Sun et al. This is an open access article distributed under the Creative Commons Attribution License, which permits unrestricted use, distribution, and reproduction in any medium, provided the original work is properly cited.

\begin{abstract}
Background. This study investigated and analyzed the current situation of sharp injuries among health care workers (HCWs) in China's Shandong Province. Methods. By means of questionnaire survey, the incidence of sharp injuries among HCWs from 36 hospitals in China's Shandong Province in October 2019 was investigated, and the results of this survey were compared with those of October 2012. Results. A total of 48165 HCWs were investigated. 549 cases of sharp injuries occurred. The incidence of sharp injuries was $1.14 \%$, which was significantly lower than that in October $2012(9.71 \%)$. In the occupational distribution of sharp injuries among HCWs, the proportion of nurses was $58.65 \%$, doctors $23.32 \%$, and interns $12.02 \%$. Among the distribution of sharp injury departments, general wards, operating rooms, intensive care units, disinfection supply centers, and outpatient clinics were the high-incidence sites of occupational exposure among HCWs. The main instruments causing sharp injuries in HCWs were syringes, scalp steel needles, surgical suture needles, vacuum blood collection needles, and glass slides. Drug administration, double-handed loop needle cap, blood extraction, surgical suture needle, and arteriovenous needle extraction were high-risk operations causing sharp instrument injuries in HCWs. Conclusion. The incidence of sharp injuries among HCWs from 36 hospitals in Shandong Province in October 2019 was significantly lower than that in October 2012. Sharp injuries were a common type of occupational exposure for HCWs. The occurrence of sharp injuries should be effectively reduced by changing wrong habitual behavior and implementing standard protective measures.
\end{abstract}

\section{Introduction}

Professional exposure to sharp injuries among HCWs refers to the occupational injuries caused by various sharp instruments such as needles, knives, and glass fragments piercing the skin during the process of medical treatment [1].

About 3.35 million HCWs suffer from sharp injuries every year around the world [2]. It has been estimated that a HCW may suffer from sharp injuries 4 times every year in Africa, the Eastern Mediterranean, and Asia [3]. The Centers for Disease Control and Prevention estimate that 385,000 sharp injuries occur among HCWs in U.S. hospitals per year [4]. The World Health Organization (WHO) estimates suggest that 1 in $10 \mathrm{HCW}$ s worldwide sustains a sharp injury each year [5]. In Egypt, a study conducted in Gharbiya
Governorate showed that $66.2 \%$ of HCWs reported that they experienced at least one sharp injury in their working life [6]. Another study which was conducted at the 3 teaching hospitals of Alexandria University reported that $67.9 \%$ of HCWs had at least 1 sharp injury in the previous 12 months [7].

The report of sharp injury is the main way for the competent department to find sharp injury events. The probability of corresponding infection is small after being stabbed by a contaminated sharp instrument [2]. Once infection occurs, the consequences are serious. Timely reporting and corresponding interventions can effectively reduce the risk of infection after exposure.

Although sharp injuries are one of the most common types of injury reported by HCWs, it is believed that they are vastly underreported [8]. Various studies indicate that 
underreporting of sharp injuries is prevalent in health care facilities worldwide, with rates of underreporting ranging from $19 \%$ to $86 \%[9,10]$. Prompt reporting of needlestick injuries is important, not only for the management of the exposure (the efficacy of PEP regimens is approximately $81 \%$ for HIV8 and $85 \%-95 \%$ for HBV 9) but also for identification of workplace hazards and evaluation of prevention measures [2].

Only a few studies have been published on sharp injuries in developing countries [11-13]. In China, HCWs are facing a high risk of sharp injuries, and the situation is grim. Although HCWs' understanding of occupational exposure has changed greatly after SARS and hospitals have also used various forms of occupational protection training and formulated corresponding systems and protective measures, still, it is difficult to resist the harm of occupational exposure to HCWs. The objectives of this study were to determine the incidence of occupational exposure and protection against sharp injuries among HCWs in Shandong Province during October 2019 and also to compare the current data with the October 2012 data.

\section{Material and Methods}

2.1. Investigation Design. An investigation about the occurrence of sharp injuries among HCWs was conducted in 36 public hospitals in China's Shandong Province in October 2019. Prior to this, the same investigation was carried out in these hospitals in October 2012.

2.2. Investigation Methods. By means of questionnaire survey, the occurrence of sharp injuries among HCWs in 36 hospitals in Shandong Province in October 2019 was investigated. The questionnaire was a slightly modified form based on the "2010 Needlestick and Sharp Object Injury Report Form" of EPINet $^{\circledR}$, which consisted of 20 variables grouped in blocks (general and individual information, the source of exposure, occurrence time, occurrence place, involved operation, occurrence department, and whether to report or not). The results of this survey were compared with those of October 2012. The two surveys were conducted in the same 36 hospitals, and all HCWs in each hospital participated in the survey. Due to the reasons of new entry, retirement, and resignation, the HCWs of each hospital changed, but the change rate was less than $3 \%$. And the scale of each hospital did not change significantly.

2.3. Sampling Method and Study Population. A clustered random sampling method was conducted to perform the questionnaire among HCWs from 36 hospitals in Shandong Province. The sample size was determined from a prevalence estimate of $10 \%$ and a population of 370,000 to give a confidence level of 0.95 and precision of 2.5 to be 554 (Epitools epidemiological calculators, Ausvet Pty Ltd., available at https://epitools.ausvet.com.au). In order to reduce the sampling error and increase the accuracy of the survey and the representativeness of the sample, we increased the sample size. 50618 questionnaires were sent out, and 48165 valid questionnaires were collected.
2.4. Informed Consent and Ethical Review. The survey was officially approved by the participating hospitals, and an agreement was signed with each hospital. The participating hospitals informed the investigated HCWs of the objectives, contents, and procedures of the survey and obtained the HCWs' consent. The survey was anonymous and did not involve the identity information of participants. This study was designed as a retrospective study, and collected data were confidentially kept. Therefore, it was exempt from ethical review.

2.5. Data Quality Control. These two investigations were both carried out with the same survey method in China's Shandong Province. Uncertain or incomplete data was eliminated to ensure the accuracy and integrity of data. The incidence of sharp injuries in this study refers to the proportion of people who suffered from sharp injuries in the last month (October 2019 or October 2012).

2.6. Statistical Analysis. Data analyses were made using SPSS Statistics V20.0. The survey results were input into SPSS 20.0 software, and the Pearson $X^{2}$ test was used to analyze the relevant data. The test level was set as alpha $=0.05$.

\section{Results}

3.1. Incidence of Sharp Injuries. A total of 48165 HCWs were surveyed. 549 cases of sharp injuries occurred. The incidence of sharp injuries was $1.14 \%$, which was significantly lower than that in $2012(9.71 \%)$. The specific data is shown in Table 1.

3.2. Profession Distribution of Sharp Injuries. Nurses (322 cases, $58.65 \%$ ) were the major components of sharp injuries, followed by doctors (128 cases, 23.32\%) and interns (66 cases, $12.02 \%)$. Incidences of sharp injuries in various occupations are shown in Table 2.

3.3. Locations of Sharp Injury Events. Professional exposure to sharp injuries may occur in all kinds of hospital environments. This survey found that general wards, operating rooms, intensive care units, disinfection supply centers, and outpatient clinics were the top five locations in which HCWs were exposed to sharp injuries. The specific data is shown in Table 3.

3.4. Tools That Caused Injuries. In the survey, we found that the top five tools causing sharp injuries were syringes, scalp steel needles, surgical suture needles, vacuum blood collection needles, and glass slides, as shown in Table 4.

3.5. Operations That Caused Sharp Injuries. In the survey, it was found that the top five operations causing sharp injuries were recapping injector needle with two hands, drug administration, drawing blood, surgical suture, and withdrawing needles, as shown in Table 5.

3.6. Report of Sharp Injuries. In the survey, the highest report rate of sharp injuries was technicians $64.71 \%$, followed by doctors $38.28 \%$, nurses $34.16 \%$, and interns $31.82 \%$. The overall report rate of sharp injuries among HCWs was 
TABle 1: The incidence of sharp injuries among HCWs in Shandong Province.

\begin{tabular}{|c|c|c|c|c|}
\hline Survey time & Survey number & Number of sharp injuries & Incidence $^{\mathrm{a}}(\%)$ & $P$ \\
\hline October 2019 & 48165 & 549 & 1.14 & \multirow{2}{*}{$<0.05$} \\
\hline October 2012 & 46600 & 4526 & 9.71 & \\
\hline
\end{tabular}

${ }^{a}$ Incidence of sharp injuries refers to the proportion of people who suffered from sharp injuries in the last month (October 2019 or October 2012). $P<0.05$ : statistically significant.

TABLE 2: Incidences of sharp injuries in various occupations in Shandong Province in October 2012 and October 2019.

\begin{tabular}{lcccccc}
\hline \multirow{2}{*}{ Profession } & \multicolumn{2}{c}{ Survey number } & \multicolumn{2}{c}{ Number of sharp injuries } & \multicolumn{2}{c}{ Incidence (\%) } \\
& October 2019 & October 2012 & October 2019 & October 2012 & October 2019 & October 2012 \\
\hline Nurse & 21953 & 21825 & 322 & 2918 & 1.47 & 13.37 \\
Doctor & 18420 & 13746 & 128 & 916 & 0.69 & 6.66 \\
Intern & 2320 & 4048 & 66 & 496 & 2.84 & $<0.05$ \\
Technician & 2043 & 1993 & 17 & 58 & 0.83 & 12.25 \\
Cleaning staff & 2578 & 2917 & 12 & 98 & 0.47 & 2.91 \\
Other & 851 & 2071 & 4 & 40 & 0.47 & 3.36 \\
Total & 48165 & 46600 & 549 & 4526 & 1.14 & $<0.05$ \\
\hline
\end{tabular}

$P<0.05$ : statistically significant.

Table 3: Locations of sharp injuries among HCWs in Shandong Province in October 2019.

\begin{tabular}{|c|c|c|c|c|c|}
\hline \multirow{2}{*}{ Department } & \multicolumn{2}{|c|}{ Number of sharp injuries } & \multicolumn{2}{|c|}{ Proportion (\%) } & \multirow{2}{*}{$P$} \\
\hline & October 2019 & October 2012 & October 2019 & October 2012 & \\
\hline General wards & 245 & 2242 & 44.63 & 49.54 & $<0.05$ \\
\hline Operating rooms & 98 & 855 & 17.85 & 18.89 & $>0.05$ \\
\hline $\mathrm{ICU}^{\mathrm{a}}$ & 37 & 236 & 6.74 & 5.21 & $>0.05$ \\
\hline $\operatorname{CSSD}^{\mathrm{b}}$ & 26 & 80 & 4.74 & 1.77 & $<0.05$ \\
\hline Outpatient dept & 23 & 116 & 4.19 & 2.56 & $<0.05$ \\
\hline Emergency dept & 21 & 121 & 3.83 & 2.67 & $>0.05$ \\
\hline Blood-drawing dept & 15 & 31 & 2.73 & 0.68 & $<0.05$ \\
\hline Medical waste repository & 14 & 43 & 2.55 & 0.95 & $<0.05$ \\
\hline Rehydration room & 10 & 101 & 1.82 & 2.23 & $>0.05$ \\
\hline Clinical laboratory & 5 & 41 & 0.91 & 0.91 & $>0.05$ \\
\hline PIVAS $^{c}$ & 4 & 38 & 0.73 & 0.84 & $>0.05$ \\
\hline Dental dept & 4 & 146 & 0.73 & 3.23 & $<0.05$ \\
\hline Hemodialysis room & 3 & 13 & 0.55 & 0.29 & $>0.05$ \\
\hline Ophthalmology dept & 3 & 34 & 0.55 & 0.75 & $>0.05$ \\
\hline Other & 41 & 429 & 7.47 & 9.48 & $>0.05$ \\
\hline Total & 549 & 4526 & 100.00 & 100.00 & \\
\hline
\end{tabular}

${ }^{\mathrm{a}} \mathrm{ICU}$ : intensive care units; ${ }^{\mathrm{b} C S S D}$ : central sterile supply department; ${ }^{\mathrm{c}}$ PIVAS: pharmacy intravenous admixture service. $P<0.05$ : statistically significant.

$35.34 \%$ in Shandong Province in October 2019, which was significantly higher than that in 2012 (15.75\%). The specific data is shown in Table 6.

\section{Discussion}

The results of this survey showed that the incidence of sharp injuries among HCWs in Shandong Province was $1.14 \%$ in October 2019, which was basically consistent with the reports in other regions of China [14-16], lower than those in other countries [17-21]. On the one hand, government and medi- cal institutions provided a lot of educational activities in recent years, the awareness of occupational exposure and protection among HCWs has increased significantly, and the protective equipment has been improved, so the incidence of sharp injuries has decreased; on the other hand, the HCWs involved in the survey failed to report or conceal sharp injury events for various reasons, which also led to a low incidence.

The incidence of sharp injuries in this survey was significantly lower than that in 2012, and incidences of sharp injuries in various occupations in October 2019 were also 
TABle 4: Tools that caused sharp injuries among HCWs in Shandong Province in October 2019.

\begin{tabular}{|c|c|c|c|c|c|}
\hline \multirow{2}{*}{ Tool } & \multicolumn{2}{|c|}{ Number of sharp injuries } & \multicolumn{2}{|c|}{ Proportion (\%) } & \multirow{2}{*}{$P$} \\
\hline & October 2019 & October 2012 & October 2019 & October 2012 & \\
\hline Syringes & 199 & 1578 & 36.25 & 34.87 & $>0.05$ \\
\hline Scalp steel needles & 110 & 1049 & 20.04 & 23.18 & $>0.05$ \\
\hline Surgical suture needles & 68 & 403 & 12.39 & 8.90 & $<0.05$ \\
\hline Blood collection needles & 26 & 90 & 4.74 & 1.99 & $<0.05$ \\
\hline Glass slides & 20 & 55 & 3.64 & 1.22 & $<0.05$ \\
\hline Vein detained needle & 17 & 141 & 3.10 & 3.12 & $>0.05$ \\
\hline Scalpel & 13 & 176 & 2.37 & 3.89 & $>0.05$ \\
\hline Sectioning knife & 6 & 44 & 1.09 & 0.97 & $<0.05$ \\
\hline Scissors & 5 & 40 & 0.91 & 0.88 & $>0.05$ \\
\hline Puncture needle & 3 & 23 & 0.55 & 0.51 & $>0.05$ \\
\hline Other & 82 & 927 & 14.94 & 20.49 & $<0.05$ \\
\hline Total & 549 & 4526 & 100.00 & 100.00 & \\
\hline
\end{tabular}

$P<0.05$ : statistically significant.

Table 5: Operations that caused sharp injuries among HCWs in Shandong Province in October 2019.

\begin{tabular}{|c|c|c|c|c|c|}
\hline \multirow{2}{*}{ Operation } & \multicolumn{2}{|c|}{ Number of sharp injuries } & \multicolumn{2}{|c|}{ Proportion (\%) } & \multirow{2}{*}{$P$} \\
\hline & October 2019 & October 2012 & October 2019 & October 2012 & \\
\hline Recapping injector needle with two hands & 90 & 467 & 16.39 & 10.32 & $<0.05$ \\
\hline Drug administration & 82 & 508 & 14.94 & 11.22 & $<0.05$ \\
\hline Drawing blood & 51 & 173 & 9.29 & 3.82 & $<0.05$ \\
\hline Surgical suture & 49 & 397 & 8.93 & 8.77 & $>0.05$ \\
\hline Withdrawing needles & 36 & 371 & 6.56 & 8.20 & $>0.05$ \\
\hline Tidying up surgical instruments & 35 & 155 & 6.38 & 3.42 & $<0.05$ \\
\hline Putting needles into the sharp box & 29 & 317 & 5.28 & 7.00 & $>0.05$ \\
\hline Preparation for infusion & 27 & 278 & 4.92 & 6.14 & $>0.05$ \\
\hline Intravenous injection & 25 & 229 & 4.55 & 5.06 & $>0.05$ \\
\hline Handling trash & 24 & 346 & 4.37 & 7.64 & $<0.05$ \\
\hline Needle or instrument delivery & 19 & 113 & 3.46 & 2.50 & $>0.05$ \\
\hline Contusion during rescue & 14 & 124 & 2.55 & 2.74 & $>0.05$ \\
\hline Intramuscular injection & 13 & 113 & 2.37 & 2.50 & $>0.05$ \\
\hline Draw arterial blood & 9 & 72 & 1.64 & 1.59 & $>0.05$ \\
\hline Surgical knife cuts & 6 & 122 & 1.09 & 2.70 & $<0.05$ \\
\hline Other & 40 & 741 & 7.29 & 16.37 & $<0.05$ \\
\hline Total & 549 & 4526 & 100.00 & 100.00 & \\
\hline
\end{tabular}

$P<0.05$ : statistically significant.

significantly lower than those in 2012. The survey results sufficiently demonstrated that the current situation of occupational exposure and protection of HCWs in Shandong Province had gradually improved.

Occupational exposure of HCWs may occur in any environment of the hospital. This current study found that general ward, operating room, and ICU were the top three departments of occupational exposure of HCWs, which was consistent with the reports in other regions of China $[14,15]$. The operations prone to sharp injuries were mainly recapping injector needle with two hands, drug administration, and drawing blood, which was consistent with the reports in China [22]. Tools that caused sharp injuries among
HCW s were mainly syringes, scalp steel needles, and surgical suture needles, which were basically consistent with the relevant reports at home and abroad $[16,17,22]$.

However, there are still some problems in occupational exposure and protection of HCWs in Shandong Province. Firstly, the use rate of safety devices is low. Since the first safety devices came out in 1988, many studies have proved that safety devices can reduce sharp injuries of HCWs [23-26]. Although there are many types of occupational exposure among HCWs, sharp injuries are the most common and harmful [27]. A survey from China Hospital Association shows that although safety devices had been gradually promoted and used throughout the country in recent years, only 
TABle 6: Report rates of sharp injuries among HCWs in Shandong Province in October 2019 and October 2012.

\begin{tabular}{lcccccrr}
\hline \multirow{2}{*}{ Profession } & \multicolumn{2}{c}{ Sharp injuries } & \multicolumn{2}{c}{ Reported number } & \multicolumn{3}{c}{ Report rate (\%) } \\
& October 2019 & October 2012 & October 2019 & October 2012 & October 2019 & October 2012 & $P$ \\
\hline Technician & 17 & 58 & 11 & 12 & 64.71 & 20.69 & $<0.05$ \\
Doctor & 128 & 916 & 49 & 152 & 38.28 & 16.59 & $<0.05$ \\
Nurse & 322 & 2918 & 110 & 430 & 34.16 & 14.73 & $<0.05$ \\
Intern & 66 & 496 & 21 & 88 & 31.82 & 17.76 & $<0.05$ \\
Other & 5 & 40 & 1 & 9 & 20.00 & 22.50 & $>0.05$ \\
Cleaning staff & 11 & 98 & 2 & 22 & 18.18 & 22.45 & $>0.05$ \\
Total & 549 & 4526 & 194 & 713 & 35.34 & 15.75 & $<0.05$ \\
\hline
\end{tabular}

$P<0.05$ : statistically significant.

$52.53 \%$ of hospitals were trying to use safety devices, and the utilization rate of safety devices is still low [22].

Secondly, the behaviors of HCWs were not standardized. Relevant studies showed that most nurses exposed to sharp injuries did not follow the relevant regulations and suggestions to prevent needlestick injuries during operation, and the incidence of sharp injuries was higher while disposing sharp instruments [28]. Gershon et al. showed that the incidence of sharp injuries was related to recapping injector needle [29]. In this survey, it was also found that recapping injector needle was a high-risk operation for sharp injuries, which was consistent with the above reports.

Thirdly, the report rate of sharp injuries is low. After occupational exposure occurs, the exposed person should report immediately and get further emergency treatment. Hospitals should establish appropriate emergency response mechanisms so that exposures can be timely reported and treated and receive regular monitoring and follow-up [30, 31]. In Salelkar et al.'s report, the report rate of sharp injuries was 32\% [32], in Khader et al.'s report 22.9\% [33], and in this survey $35.34 \%$, which is basically the same as the above reports. The most common reason for failing to report is the lack of awareness among HCWs [22].

In the case of sharp instrument injury contaminated by blood-borne pathogens, the corresponding infection rate is estimated to be as follows: hepatitis B 3-10\%; hepatitis C 0.8-3\%; and HIV 0.3\% [34]. Hospitals should establish corresponding emergency response mechanisms so that exposures can be reported and dealt with in a timely manner. In view of the risk of postsharp injury infection, medical and health institutions should assess the risk of infection based on the available information, including the results of examinations of the source patients and types and duration of occupational exposure; assess and determine the level of exposure and the level of infectious pathogenicity of the source of exposure; and determine whether to use preventive drugs and what preventive measures to take. The case should have been followed up. In view of the psychological barriers of HCWs after sharp injuries [35], we should also pay attention to the changes of psychological status of HCWs and provide timely psychological support and intervention to ensure the physical and mental health of HCWs.

\section{Conclusion and Limitations}

There were some limitations to this study. First, it relied on the respondents' questionnaire data, which were subject to subjective consciousness and recall bias. This could have resulted in an underestimation of actual incidence of sharp injuries. In addition, the incidence of sharp injuries could vary among hospitals due to the difference in safety measures, reporting systems, and facility appliances. Due to the limitation of the survey content, it was difficult to compare and analyze the differences among these hospitals.

The incidence of sharp injuries among HCWs from 36 hospitals in Shandong Province in October 2019 was significantly lower than that in October 2012. Sharp injuries were a common type of occupational exposure for HCWs. The occurrence of sharp injuries should be effectively reduced by changing wrong habitual behavior and implementing standard protective measures.

\section{Data Availability}

The data used to support the findings of this study are included within the article.

\section{Conflicts of Interest}

The authors declare that they have no conflicts of interest regarding the publication of this paper.

\section{Acknowledgments}

This work was supported by the Nosocomial Infection Control Center of Shandong Provincial. The authors thank the participants of the 36 surveyed hospitals for their agreement to take part in the study and their contribution to the survey data collection.

\section{References}

[1] Centers for Disease Control and Prevention, "The National Institute for Occupational Safety and Health (NIOSH) [OL].[2018-07-09]," March 2018, https://www.cdc.gov/niosh/ stopsticks/sharp-injuries.html.

[2] A. Pruss-Ustun, E. Rapiti, and Y. Hutin, "Estimation of the global burden of disease attributable to contaminated sharps 
injuries among health-care workers," American Journal of Industrial Medicine, vol. 48, no. 6, pp. 482-490, 2005.

[3] S. Q. Wilburn and G. Eijkemans, "Preventing needlestick injuries among healthcare workers: a WHO-ICN collaboration," International Journal of Occupational and Environmental Health, vol. 10, no. 4, pp. 451-456, 2004.

[4] A. L. Panlilio, J. G. Orelien, P. U. Srivastava et al., "Estimate of the annual number of percutaneous injuries among hospitalbased healthcare workers in the United States, 1997-1998," Infection Control and Hospital Epidemiology, vol. 25, no. 7, pp. 556-562, 2004.

[5] A. Arafa and A. Mohammed, "Injection practices among health care workers and risk factor for hepatitis B virus in a governmental hospital," Medical Journal of Cairo University, vol. 80, pp. 191-196, 2012.

[6] N. A. Ismail, A. M. A. Ftouh, W. H. El Shoubary, and H. Mahaba, "Safe injection practice among health-care workers in Gharbiya Governorate, Egypt," EMHJ-Eastern Mediterranean Health Journal, vol. 13, no. 4, pp. 893-906, 2007.

[7] M. I. Hanafi, A. M. Mohamed, M. S. Kassem, and M. Shawki, "Needlestick injuries among health care workers of University of Alexandria hospitals," Eastern Mediterranean Health Journal, vol. 17, no. 1, pp. 26-35, 2011.

[8] G. Kassa, D. Selenic, M. Lahuerta et al., "Occupational exposure to bloodborne pathogens among health care workers in Botswana: reporting and utilization of postexposure prophylaxis," American Journal of Infection Control, vol. 44, no. 8, pp. 879-885, 2016.

[9] Centers for Disease Control and Prevention (CDC), "Updated U.S. Public Health Service guidelines for the management of occupational exposures to HBV, HCV, and HIV and recommendations for postexposure prophylaxis," MMWR. Morbidity and Mortality Weekly Report, vol. 50, pp. 1-33, 2001.

[10] O. S. Kim, J. S. Jeong, K. M. Kim et al., "Underreporting rate and related factors after needlestick injuries among healthcare workers in small or medium-sized hospitals," Korean Journal of Nosocomial Infection Control, vol. 16, pp. 29-36, 2011.

[11] E. M. Mbaisi, Z. Ng'ang'a, P. Wanzala, and J. Omolo, "Prevalence and factors associated with percutaneous injuries and splash exposures among health-care workers in a provincial hospital, Kenya, 2010," Pan African Medical Journal, vol. 14, p. 10, 2013.

[12] W. G. Li, H. Xu, Z. QF, L. Jia, and A. M. Gu, "Current status of sharp instrument injuries among medical staff of 28 hospitals in Shandong province," Chinese Journal of Infection Control, vol. 23, no. 7, pp. 1605-1607, 2013.

[13] A. A. Reda, S. Fisseha, B. Mengistie, and J.-M. Vandeweerd, "Standard precautions: occupational exposure and behavior of health care workers in Ethiopia," PLoS One, vol. 5, no. 12, article e14420, 2010.

[14] H. Zhang, Y. Yang, L. Cai, X. U. Feihua, J. Wang, and X. Liu, "Survey on the current status of sharp injury among 11358 healthcare workers in Gansu Province," Chinese Journal of Infection Control, vol. 13, no. 9, pp. 548-551, 2014.

[15] X. U. Shilan, W. U. Xiyi, R. A. Li et al., "A current situation survey on sharp injury in 840 medical workers," Chinese Journal of Evidence-Based Medicine, vol. 11, no. 6, pp. 636-639, 2011.

[16] H. Zhang, Y. Zhang, L. Cai et al., "Current status and causes of sharp injuries among healthcare workers in hospials of Chinese westernareas," Chinese Journal of Nosocomiology, vol. 28, no. 18, pp. 2876-2880, 2018.
[17] S. Wicker, J. Jung, R. Allwinn, R. Gottschalk, and H. F. Rabenau, "Prevalence and prevention of needlestick injuries among healthcarewokers in a German university hospital," International Archives of Occupational and Environmental Health, vol. 81, no. 3, pp. 347-354, 2008.

[18] J. D. Brewer, D. M. Elston, A. T. Vidimos, S. A. Rizza, and S. J. Miller, "Managing sharps injuries and other occupational exposures to HIV, HBV, and HCV in the dermatology office," Journal of the American Academy of Dermatology, vol. 77, no. 5, pp. 946-951.e6, 2017.

[19] T. Grimmond and L. Good, "Exposure Survey of Trends in Occupational Practice (EXPO-S.T.O.P.) 2015: a national survey of sharps injuries and mucocutaneous blood exposures among health care workers in US hospitals," American Journal of Infection Control, vol. 45, no. 11, pp. 1218-1223, 2017.

[20] N. T. Sharew, G. B. Mulu, T. D. Habtewold, and K. D. Gizachew, "Occupational exposure to sharps injury among healthcare providers in Ethiopia regional hospitals," Annals of Occupational and Environmental Medicine, vol. 29, no. 1, p. 7, 2017.

[21] C. Matsubara, K. Sakisaka, V. Sychareun, A. Phensavanh, and M. Ali, "Prevalence and risk factors of needle stick and sharp injury among tertiary hospital workers, Vientiane, Lao PDR," Journal of Occupational Health, vol. 59, no. 6, pp. 581-585, 2017.

[22] J. Sun, X. U. Hua, G. U. Anman et al., "Occupational exposure and protection among health care workers in China," Chinese Journal of Infection Control, vol. 15, no. 9, p. 6818, 2016.

[23] D. M. Bell, "Occupational risk of human immunodeficiency virus infection in healthcare workers: an overview," The American Journal of Medicine, vol. 102, no. 5, pp. 9-15, 1997.

[24] D. Adams and T. S. Elliott, "Impact of safety needle devices on occupationally acquired needlestick injuries: a four-year prospective study," The Journal of Hospital Infection, vol. 64, no. 1 , pp. 50-55, 2006.

[25] J. Jagger, J. Perry, A. Gomaa, and E. K. Phillips, “The impact of U.S. policies to protect healthcare workers from bloodborne pathogens: the critical role of safety-engineered devices," Journal of Infection and Public Health, vol. 1, no. 2, pp. 62-71, 2008.

[26] F. Lamontagne, D. Abiteboul, I. Lolom et al., "Role of safetyengineered devices in preventing needlestick injuries in 32 French hospitals," Infection Control and Hospital Epidemiology, vol. 28, no. 1, pp. 18-23, 2007.

[27] E. Cho, H. Lee, M. Choi, S. H. Park, I. Y. Yoo, and L. H. Aiken, "Factors associated with needlestick and sharp injuries among hospital nurses: a cross-sectional questionnaire survey," International Journal of Nursing Studies, vol. 50, no. 8, pp. 10251032, 2013.

[28] A. C. Weltman, L. J. Short, M. H. Mendelson, D. E. Lilienfeld, and M. Rodriguez, "Disposal-related sharps injuries at a New York City teaching hospital," Infection Control and Hospital Epidemiology, vol. 16, no. 5, pp. 268-274, 1995.

[29] R. R. Gershon, J. M. Pearson, M. F. Sherman, S. M. Samar, A. N. Canton, and P. W. Stone, "The prevalence and risk factors for percutaneous injuries in registered nurses in the home health care sector," American Journal of Infection Control, vol. 37, no. 7, pp. 525-533, 2009.

[30] B. Green and E. C. Griffiths, "Psychiatric consequences of needlestick injury," Occupational Medicine, vol. 63, no. 3, pp. 183$188,2013$. 
[31] S. Wicker, A. V. Stirn, H. F. Rabenau, L. von Gierke, S. Wutzler, and C. Stephan, "Needlestick injuries: causes, preventability and psychological impact," Infection, vol. 42, no. 3, pp. 549-552, 2014.

[32] S. Salelkar, D. D. Motghare, and M. S. Kulkami, "Study of needle stick injuries among health care workers at a tertiary care hospital," Indian Journal of Public Health, vol. 54, no. 1, pp. 18-20, 2010.

[33] Y. Khader, S. Burgan, and Z. Amarin, "Self-reported needlestick injuries among dentists in north Jordan," Eastern Mediterranean Health Journal, vol. 15, no. 1, pp. 185-189, 2009.

[34] World Health Organization, WHO Best Practices for Injections and Related Procedures Toolkit, WHO, Geneva, Switzerland, 2010.

[35] S. H. Naghavi, O. Shabestari, and J. Alcolado, "Post-traumatic stress disorder in trainee doctors with previous needlestick injuries," Occupational Medicine, vol. 63, no. 4, pp. 260-265, 2013. 